\title{
A sample of southern Compact Steep Spectrum radio sources: The VLBI observations *
}

\author{
A. Tzioumis ${ }^{1}$, E. King ${ }^{6,11}$, R. Morganti ${ }^{3}$, D. Dallacasa ${ }^{2,9}$, C. Tadhunter ${ }^{4}$, C. Fanti ${ }^{2,10}$, J. Reynolds ${ }^{1}$, D. Jauncey ${ }^{1}$, \\ R. Preston ${ }^{5}$, P. McCulloch ${ }^{6}$, S. Tingay ${ }^{1}$, P. Edwards ${ }^{7,12}$, M. Costa $^{6}$, D. Jones ${ }^{5}$, J. Lovell ${ }^{6,1}$, R. Clay ${ }^{7}$, D. Meier ${ }^{5}$, \\ D. Murphy ${ }^{5}$, R. Gough ${ }^{1}$, R. Ferris ${ }^{1}$, G. White ${ }^{8}$, and P. Jones ${ }^{8}$
}

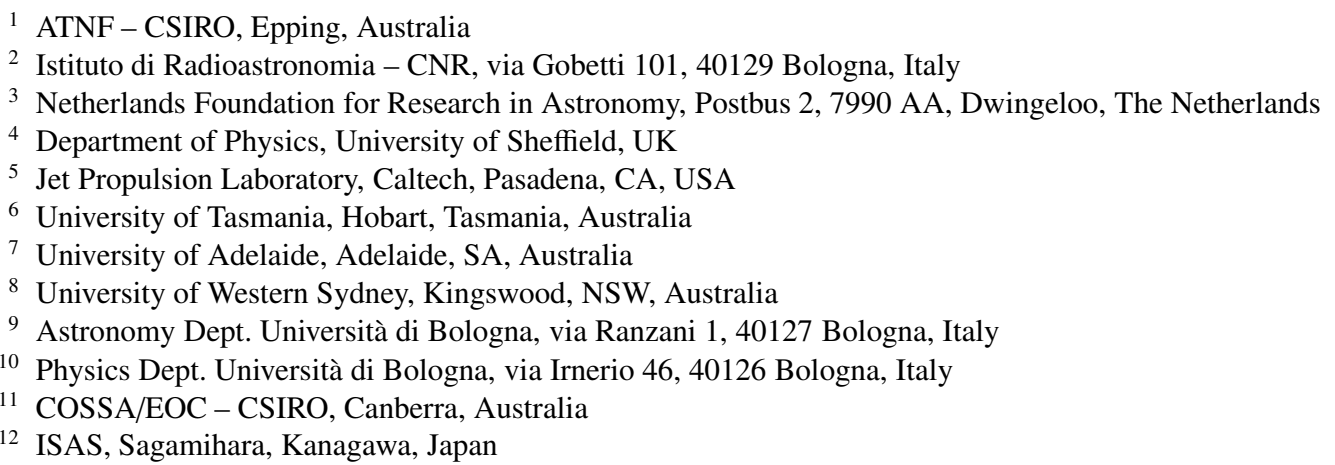

Received 25 April 2002 / Accepted 26 June 2002

\begin{abstract}
A small sample of 7 southern Compact Steep Spectrum (CSS) radio sources has been selected as part of the study of a larger flux-limited complete sample of radio sources. High resolution images, using the VLBI network in the southern hemisphere and the high resolution MERLIN array, are presented for all sources in the CSS sample. The overall morphology of each source consists of well-defined double lobes but with substantial diffuse and extended components present. In the majority of cases only a fraction of the total flux density is detected on the VLBI baselines, indicating the presence of larger extended radio structures. However, all sources are unresolved at arcsecond scales and are of sub-galactic size, with linear size in the range $0.1-2 \mathrm{kpc}$. The radio properties of the sources agree well with CSS sources in other samples.
\end{abstract}

Key words. galaxies: active - radio continuum: general

\section{Introduction}

Among the many open questions about powerful radiogalaxies is the early evolution of these objects. This is an important issue not only from the point of view of the detailed phenomenology of these objects but also for understanding the evolution of massive galaxies. Galaxies in their early stage of radio activity are likely to have their nuclear regions enshrouded in a cocoon or thick disk of material left over from the event(s) that trigger the activity. The radio jet expanding in this medium will interact and sweep out this rich interstellar medium (ISM). These processes have been suggested to profoundly affect even the star formation history in luminous galaxies (Silk \& Rees 1998). Thus, the study of the early-phase of radio activity and its effect on the galaxy medium has broad implications.

\footnotetext{
Send offprint requests to: A. Tzioumis

* Based on observations with the Southern Hemisphere VLBI Network (SHEVE) and the MERLIN.
}

Compact Steep Spectrum (CSS) and Gigahertz Peaked Spectrum (GPS) radio sources have now been recognised to be the likely candidates of radio sources in such an early stage of their life. These radio sources are powerful $\left(P_{2.7 \mathrm{GHz}} \gtrsim 10^{25.5} \mathrm{~W} \mathrm{~Hz}{ }^{-1}\right)$, have a steep spectral index at high frequencies $\left(\alpha<-0.5, S \sim v^{\alpha}\right)$ and sub-galactic size $(<20 \mathrm{kpc})$. Indeed, the turnover often observed in their spectra (around $1 \mathrm{GHz}$ for the GPSs and about $100 \mathrm{MHz}$ for the CSSs) is usually interpreted as synchrotron self-absorption, with the frequency of this turnover varying with the linear scale of the source, although free-free absorption may also play a role (Bicknell et al. 1997). A review of the characteristics of $\mathrm{CSS} / \mathrm{GPS}$ sources at different wavelengths is given in O'Dea (1998).

The age of CSS/GPS sources has been recently estimated using both the lobe proper motion (e.g. Owsianik \& Conway 1998) and the radiative ages (Murgia et al. 1999). The ages derived are less than a few thousand years, therefore strongly supporting the "youth" scenario in the interpretation of these 
sources. A summary of the results is given in the review by Fanti et al. (2000).

The sub-galactic size and the young age of these sources makes them an extremely interesting class of radio source. They can be used to probe further the characteristics of the ISM in the early phase of radio source evolution and the effects of the radio plasma expanding in this medium. This has been done in a restricted number of objects both using the atomic hydrogen (e.g. Conway 1996; Pilström 2001; Morganti et al. 2001; Peck \& Taylor 2001) and the ionised gas (e.g. Gelderman \& Whittle 1994; Morganti et al. 1997; Axon et al. 2000; O’Dea et al. 2002).

An other important aspect in the study of these objects is the comparison of their characteristics with those of the extended radio galaxies. This has been done quite extensively in the radio to investigate the evolutionary scenario. At wavelengths different from radio the available data are still limited. ISO observations (Fanti et al. 2000) and optical and near IR data (de Vries et al. 1998) showed no significant difference between CSS/GPS sources and extended radio galaxies with similar radio power, failing therefore to detect any extra component capable to "frustrate" the CSS/GPS sources.

To extend the comparison between the optical properties of CSS/GPS and extended radio sources, a group of CSS/GPS sources was selected from a complete sub-sample of the $2 \mathrm{Jy}$ sample of radio sources (see Sect. 2 for details). Optical spectra were obtained for both the compact as well as for the extended sources in this sample (see Tadhunter et al. 1993) and the spectral characteristics could therefore be compared for objects in a similar range of redshift and radio power. A first-order similarity was found between the spectra of CSS/GPS sources and the extended source of similar radio power although the former may be of slightly lower ionisation, possible evidence for the compression effect of shocks due to jet/cloud interaction (Morganti et al. 1997). For the 2 Jy CSS/GPS source sample however, there is a lack of high resolution radio images capable of providing information on their morphology. Most of the well studied CSS/GPS galaxies tend to have symmetric, double-lobed radio structures (e.g. Dallacasa et al. 1995; O'Dea 1998), reminiscent of scale-down versions of extended, edge-brightened Fanaroff-Riley II sources. Collecting this information for the CSS/GPS sources in the $2 \mathrm{Jy}$ sample is particularly important also for the interpretation of their optical data. The presence of more complex radio morphology in these sources (or in some of them) would be an indication of a particularly strong interaction between the radio plasma and the ISM, more than usually found in this type of radio source.

In order to fill this gap, in this paper we present and discuss new high resolution (VLBI and MERLIN) data for this group of seven 2 Jy CSS/GPS sources. These radio images permit a proper morphological classification of the sub-kpc radio emission in these objects.

\section{The Sample}

The group of CSS/GPS sources considered here has been selected from the complete sub-sample of the Wall \& Peacock (1985) (WP85) 2 Jy sample described in

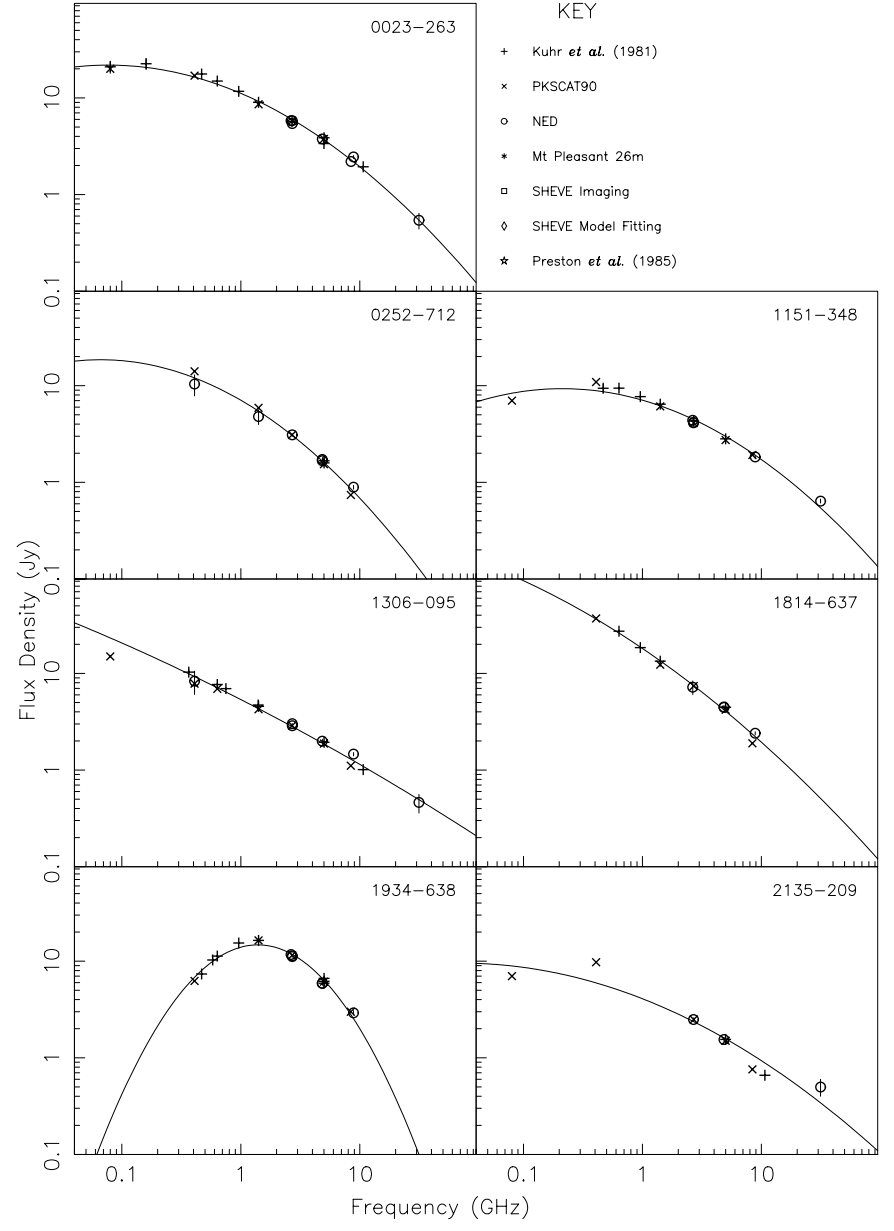

Fig. 1. Radio spectral index plots for all sources.

Table 1. CSS sources in the sample with a summary of their characteristics.

\begin{tabular}{lcccccc}
\hline \hline Name & ID & $m_{V}$ & $z$ & $\begin{array}{c}\text { scale } \\
\text { pc/mas }\end{array}$ & $\begin{array}{c}\log P_{2.7 \mathrm{GHz}} \\
\mathrm{W} \mathrm{Hz}^{-1}\end{array}$ & $\alpha_{2.7}^{8.4}$ \\
\hline $0023-263$ & $\mathrm{G}$ & 19.5 & 0.322 & 2.9 & 26.91 & -0.8 \\
$0252-712$ & $\mathrm{G}$ & 20.9 & 0.566 & 3.7 & 27.28 & -1.25 \\
$1151-348$ & $\mathrm{Q}$ & 17.8 & 0.258 & 2.5 & 26.56 & -0.7 \\
$1306-095$ & $\mathrm{G}$ & 20.5 & 0.464 & 3.5 & 26.94 & -0.85 \\
$1814-637$ & $\mathrm{G}$ & 18.0 & 0.063 & 0.8 & 25.54 & -1.2 \\
$1934-638^{*}$ & $\mathrm{G}$ & 18.4 & 0.183 & 2.0 & 26.68 & -1.15 \\
$2135-209$ & $\mathrm{G}$ & 19.4 & 0.635 & 3.9 & 27.20 & -1.05 \\
\hline
\end{tabular}

${ }^{*}$ Gigahertz-Peaked Spectrum source (GPS).

Tadhunter et al. (1993). This sub-sample is formed by 88 radio sources (radio galaxies and quasars) having $\mathrm{S}_{2.7 \mathrm{GHz}}>2.0 \mathrm{Jy}$, $\delta<10^{\circ}$ and $z<0.7$. The sample has been studied extensively at various wavelengths: optical (Tadhunter et al. 1993, 1998), radio (Morganti et al. 1993, 1997, 1999; Venturi et al. 2000) and X-ray (Siebert et al. 1996; Padovani et al. 1999; Trussoni et al. 1999).

According to the definition in Fanti et al. 1995, a source is classified as CSS when at high frequency $\alpha<-0.5\left(S \sim v^{\alpha}\right)$ and the linear dimensions are $\leq 15 \mathrm{kpc}^{1}$. To make the selection

${ }^{1} H_{0}=100 \mathrm{~km} \mathrm{~s}^{-1} \mathrm{Mpc}^{-1}, q_{0}=0.5$. 


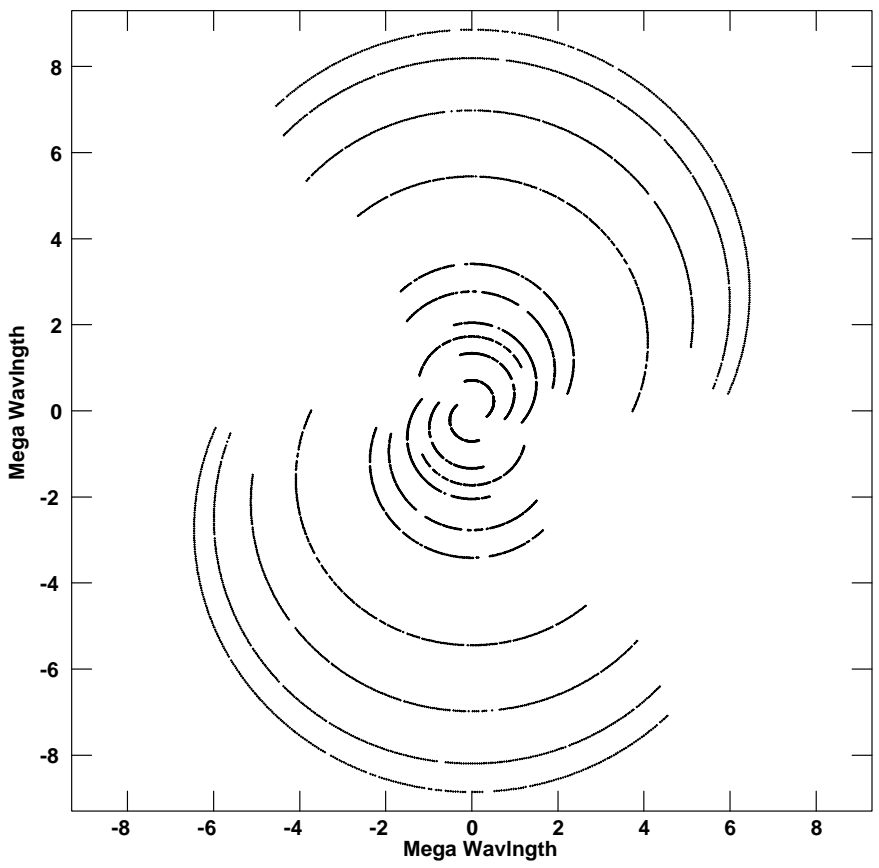

Fig. 2. Typical uv coverage of the SHEVE array, from the 0252-712 at $2.3 \mathrm{GHz}$. (No detections to Perth or Hartebeesthoek).

consistent with previous works of Fanti et al. (1990) and Spencer et al. (1991), only objects with $P_{2.7 \mathrm{GHz}}>$ $10^{25.5} \mathrm{~W} \mathrm{~Hz}^{-1}$ were selected. With these criteria we find $7 \mathrm{CSS}$ (6 galaxies and 1 quasar) in our complete sample i.e. $8 \%$ of the total number of sources or $12.5 \%$ of the $P_{2.7 \mathrm{GHz}}>$ $10^{25.5} \mathrm{~W} \mathrm{~Hz}^{-1}$ sub-sample.

The seven CSS sources in our sample are listed in Table 1 together with some additional information. Their radio power is consistent with the typical radio power for this class of objects. Optical identifications and redshifts (WP85; di Serego Alighieri et al. 1994) are available for every source in this sample. All sources with declination north of $-40^{\circ}$ are unresolved in VLA images $\left(\theta<3^{\prime \prime}\right)$ and the remaining are part of the ATCA calibration list $\left(\theta<1^{\prime \prime}\right)$. Radio spectra from the present radio observations and from data in the literature (King 1994) are presented in Fig. 1. The two-point spectral index $\alpha_{2.7}^{8.4}$ listed in Table 1 clearly demonstrates the steep spectrum nature of these objects.

\section{Observations, data reduction and analysis}

The VLBI observations of all sources were made with the SHEVE network (Preston et al. 1993; Jauncey et al. 1994) between April 1991 and February 1993, using the MK2 recording system (Clark 1973). The characteristics of the antennas and receivers in the SHEVE network are listed in Table 2. The details of each observation are given in Table 3.

All sources were observed at $2.3 \mathrm{GHz}$, but for 2135-209 only a single baseline (Tidbinbilla-Hobart) was obtained in a 12 hour observation. The sources $0023-263,1151-348$ and 1934-638 were also observed at $8.4 \mathrm{GHz}$ and 1934-638 at $4.9 \mathrm{GHz}$ as well. The GPS source 1934-638 was observed as part of another program and the details of the observations and results are presented elsewhere (King 1994; Tzioumis et al. 1996) while some discussion of the source properties is presented here.

The tapes recorded at each station were processed at the Caltech-JPL Block2 correlator in Pasadena and the NRAO AIPS package was used for global fringe fitting. The amplitude calibration and editing of the visibilities were subsequently performed using the Caltech VLBI package. Self-calibration and imaging of the data were performed using DIFMAP (Shepherd et al. 1994) and the AIPS packages. The single-baseline data for 2135-209 was used to obtain a simple model of the source using the program MODELFIT from the Caltech VLBI package.

On the very long baselines from the antennas at eastern Australia (ATNF, DSN, Hobart) to Perth $(3000 \mathrm{~km})$ and Hartebeesthoek (9500 km), only 1934-638 and VLBI calibrator sources were detected, i.e. all other sources are completely resolved out at these spacings and sensitivities. This implies that there are no very compact components $(\theta \ll 3$ mas) with flux density larger than about $40 \mathrm{mJy}$, the detection limit from the intercontinental baselines.

A "typical" uv-coverage from the SHEVE array is presented in Fig. 2, for the source 0252-712 at 2.3 GHz. Note that all sources except 1934-638, were not detected on the long baselines to Perth or Hartebeesthoek and uv-tracks are not shown.

The limited number of antennas in the network and the lack of short baselines limit the dynamic range achievable from these observations. In particular, for many of the sources the total flux density in the VLBI images is significantly lower than that measured with lower resolution observations. This implies that low surface brightness extended structures with angular sizes between 0.1 and 1 arcsec are often present but invisible by the VLBI interferometers, as discussed in the next section on each source.

For 0023-263 and 2135-209 we also present MERLIN observations at $5.0 \mathrm{GHz}$, which provide information on more extended structures, not detected by the VLBI observations.

The new images of the six sources observed in this program are presented in Figs. 3 through 9. Restoring beams together with the noise measure on the image plane are given in Table 3.

The overall size of most sources in this sample was not previously well determined at radio wavelengths. The largest angular/linear size for each source was estimated from the images, adopting a "low resolution" approach (cf. Dallacasa et al. 1995). Most of the sources show distinct and well separated "lobes", and extended structure can be seen in most of them. Their sizes and flux densities were estimated from the images and are summarised in Table 5. The component angular dimensions were generally estimated directly from the images (except were otherwise stated) by considering the lowest reliable contour; they are given as full-width axis sizes. This approach was adopted because almost all components show extended structures and it is then difficult to fit single Gaussian components. The measurements provided values well in excess of the beam size, and they were not deconvolve given they would not change significantly; therefore, all sizes in Table 5 should be treated as upper limits. 
Table 2. Antennas participating in the VLBI observations. Note that the three Tidbinbilla antennas are at essentially the same location and only one antenna is used at any one time, depending on availability. The SEFD (system equivalent flux density) is given by the ratio between system temperature $\left({ }^{\circ} \mathrm{K}\right)$ and gain ${ }^{\circ} \mathrm{K} / \mathrm{Jy}$.

\begin{tabular}{|c|c|c|c|c|}
\hline \multirow[t]{2}{*}{ Key } & \multirow[t]{2}{*}{ Antenna } & \multirow{2}{*}{$\begin{array}{r}\text { Size } \\
(\mathrm{m})\end{array}$} & \multicolumn{2}{|c|}{ "SEFD (Jy) } \\
\hline & & & $2.3 \mathrm{GHz}$ & $8.4 \mathrm{GHz}$ \\
\hline $\mathrm{P}$ & Parkes(ATNF) & 64 & 90 & 90 \\
\hline M & Mopra (ATNF)* & 22 & 400 & 400 \\
\hline $\mathrm{C}$ & Narrabri-CA (ATNF) & 22 & 400 & 400 \\
\hline 3 & Tidbinbilla (DSS43) & 70 & 15 & 20 \\
\hline 5 & Tidbinbilla (DSS45) & 34 & 165 & 130 \\
\hline 2 & Tidbinbilla (DSS42) & 34 & 100 & 130 \\
\hline $\mathrm{H}$ & Hobart (Univ.Tasmania) & 26 & 750 & 650 \\
\hline $\mathrm{G}$ & Perth (ESA) & 15 & 3300 & - \\
\hline $\mathrm{E}$ & Harteebeesthoek & 26 & 400 & 950 \\
\hline
\end{tabular}

* The Mopra antenna became operational in late 1991.

Table 3. Observations and images presented in this paper. The dynamic range indicates the ratio of the peak brightness in the image to the peak in the residual map. The $\sigma_{\text {rms }}$ entry refers to the residual rms noise in regions devoid of any source emission. Entries in the last column refer to the figure in which each image appears.

\begin{tabular}{cccclccccc}
\hline \hline Source & Epoch & $\begin{array}{c}\text { Freq. } \\
\text { GHz }\end{array}$ & $\begin{array}{c}\text { Duration } \\
\text { (hours) }\end{array}$ & Stations & $\begin{array}{c}B_{\max } * \\
M \lambda\end{array}$ & $\begin{array}{c}\text { Beam } \\
\text { mas }\end{array}$ & $\begin{array}{c}\text { Dynamic } \\
\text { Range }\end{array}$ & $\begin{array}{c}\sigma \\
\text { mJy/beam }\end{array}$ & $\begin{array}{c}\text { Figure } \\
\text { Ref. }\end{array}$ \\
\hline 0023-263 & 1991.34 & 2.29 & 11.5 & 32PHC & 10.5 & $31 \times 14-82$ & $60: 1$ & 4.5 & 3 \\
& 1991.90 & 8.42 & 11 & 32PHCM & 29.9 & $13 \times 5-77$ & $40: 1$ & 1.7 & 3 \\
& 1992.05 & 4.99 & 6 & MERLIN & 3.6 & $168 \times 86-2$ & $130: 1$ & 3.5 & 4 \\
$0252-712$ & 1993.14 & 2.29 & 10.25 & 2PHCMGE & 8.9 & $32 \times 19-73$ & $65: 1$ & 8 & 5 \\
$1151-348$ & 1991.34 & 2.29 & 11.75 & 35PHC & 10.7 & $29 \times 1389$ & $70: 1$ & 4.5 & 6 \\
& 1992.24 & 8.42 & 11 & 35PHCM & 30.3 & $11 \times 5-81$ & $35: 1$ & 3.6 & 6 \\
$1306-095$ & 1993.14 & 2.29 & 10.5 & 32PHCGE & 10.7 & $48 \times 15-73$ & $20: 1$ & 5.7 & 7 \\
$1814-637$ & 1993.14 & 2.29 & 12 & 32PHCMGE & 9.5 & $32 \times 17-86$ & $50: 1$ & 5.8 & 8 \\
$1934-638$ & & $2.29,4.85 \mathcal{E}$ 8.42 GHz images in King (1994) E Tzioumis et al. $(1996)$ & & 10 \\
$2135-209$ & 1991.55 & 2.29 & 12 & 3H & 3.6 & Modelfit only & & & \\
& 1992.91 & 4.99 & 6.25 & MERLIN & 3.6 & $163 \times 3410$ & $130: 1$ & 2.3 & 9 \\
\hline
\end{tabular}

* Maximum baseline with significant dectection of each source. No detections to Perth (G) or Hartebeesthoek (E).
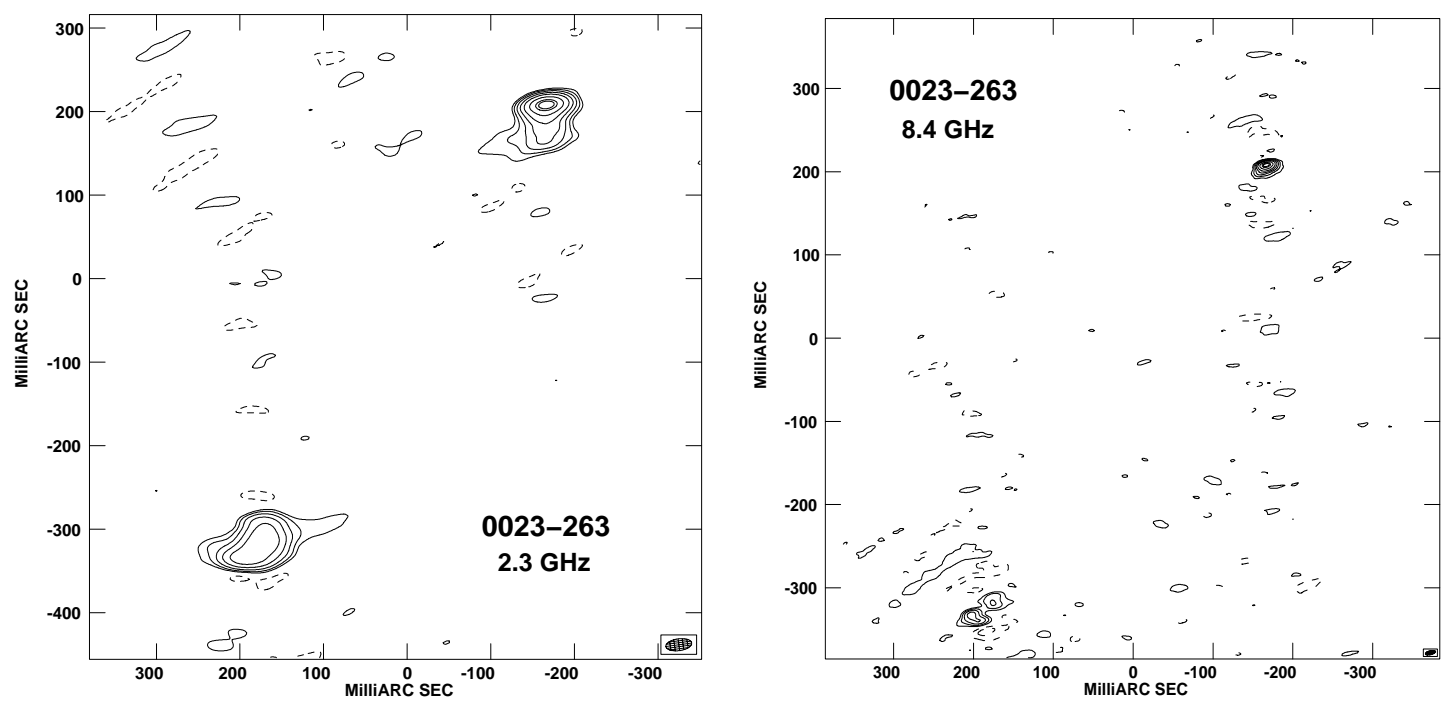

Fig. 3. (Left) PKS 0023-263 at $2291 \mathrm{MHz}$ from the SHEVE array. The peak level is $1.07 \mathrm{Jy} / \mathrm{beam}$ and contours are shown at $-2,-1,1,2,4,8$, $16,35,65,80 \%$ of the peak. (Right) PKS 0023-263 at $8419 \mathrm{MHz}$ from the SHEVE array. The peak level is $0.41 \mathrm{mJy} /$ beam and contours are shown at $-2,-1,1,2,4,8,16,35,65,80 \%$ of the peak. 


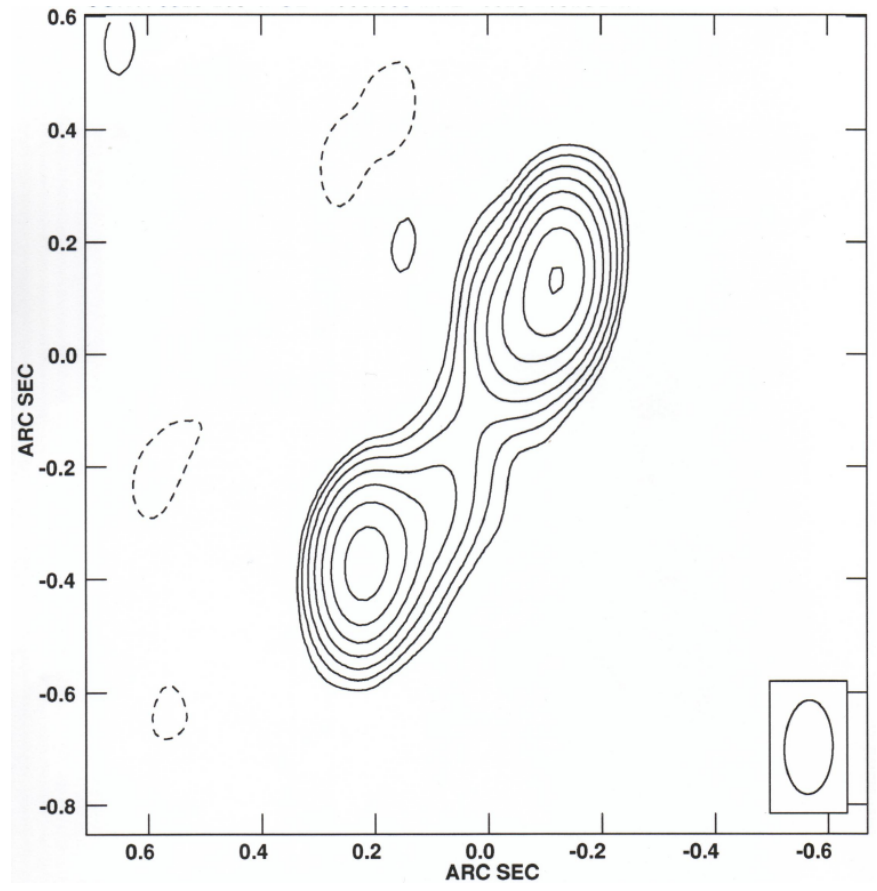

Fig. 4. PKS 0023-263 at $4996 \mathrm{MHz}$ from the MERLIN array. The peak level is $1.41 \mathrm{Jy} /$ beam and contours are shown at $-6,-3,-1.5,-0.75$, $0.75,1.5,3,6,12,24,48,96 \%$ of the peak.

Table 4. Overall angular and linear sizes of the sample sources and separation of strongest components in the two lobes.

\begin{tabular}{lccccc}
\hline \hline \multirow{2}{*}{ Name } & & \multicolumn{4}{c}{ Component separation } \\
& $\theta_{\max }$ & Lin. size & $\theta$ & $l$ & Orientation \\
& $\operatorname{mas}$ & $\mathrm{pc}$ & $\mathrm{mas}$ & $\mathrm{pc}$ & $\circ$ \\
\hline $0023-263$ & 680 & 1970 & 654 & 1900 & -34 \\
$0252-712$ & 240 & 900 & 145 & 540 & 7 \\
$1151-348$ & 170 & 425 & 91 & 228 & 72 \\
$1306-095$ & 460 & 1600 & 373 & 1290 & -41 \\
$1814-637$ & 410 & 328 & 239 & 191 & -20 \\
$1934-638^{*}$ & 70 & 140 & 42 & 84 & 89 \\
$2135-209$ & $\sim 250$ & $\sim 1000$ & 167 & 650 & 52 \\
\hline
\end{tabular}

${ }^{*}$ Tzioumis et al. (1996).

The separation between the strongest component in each of the lobes was also measured from the images. This separation is a very sensitive parameter since it is well determined by the "beating" in the visibilities and can be used to search for any source expansion in time, even with sparse uv coverage. The results are presented in Table 4.

For the two sources observed at 2.3 and $8.4 \mathrm{GHz}$, a twopoint spectral index for each lobe has also been determined.

\section{Comments on individual sources}

In the following, a description of the morphology of each source is given:

0023-263 This radio source has been identified with a galaxy of $m_{v}=19.5$ by WP85 at a redshift of 0.322 (Tadhunter et al. 1993; di Serego Alighieri et al. 1994). The VLBI images at 2.3 and $8.4 \mathrm{GHz}$ are shown in Fig. 3.
At both VLBI frequencies this radio galaxy shows a basic double structure with a separation of $\sim 650$ mas $(\sim 1.9 \mathrm{kpc})$ in PA $-34^{\circ}$ between the peak brightness of each lobe. Both radio emitting regions are complex and extended structures are evident. In particular, the south-eastern component is resolved into two sub-components embedded into more diffuse emission. Thus, it is not possible to fit simple Gaussian components, and sizes for each lobe are estimated from the images. Both lobes are less than $\sim 100$ mas $(\sim 0.3 \mathrm{kpc})$ in extent and the south-eastern one has an overall spectral index $(\alpha=-1.3)$ steeper than the north-western component ( $\alpha=-0.7)$, possibly also because it is more resolved.

The flux density detected in the VLBI images accounts for only about $40 \%$ of the total source flux density at each frequency. This indicates that there is undetected extended scale structures in the range 0.1 to 1 arcsec. The same fraction of flux density is undetected at both frequencies, suggesting that the underlying diffuse structure has similar spectral index $(\sim-0.9)$ to the components visible in the images. No flat spectrum component indicative of a core is detected between the lobes at the detection limits $(5 \sigma)$ of $\sim 20 \mathrm{mJy} /$ beam at $2.3 \mathrm{GHz}$ and $\sim 10 \mathrm{mJy} /$ beam at $8.4 \mathrm{GHz}$. We decided to use $5 \sigma$ as detection limit for the cores (in this and in the following sources), given that, the complexity of the radio emission in relation to the limited uv-sampling, increases the incidence of clean artifacts at lower levels.

The MERLIN image at $5 \mathrm{GHz}$ (Fig. 4) with much lower resolution shows the basic double-lobe structure of the source and the extended emission surrounding the lobes. About $90 \%$ of he total flux density is detected (3.4 Jy out of $3.8 \mathrm{Jy}$ ) indicating little extended emission at arcsecond scales.

0252-712 This object has been identified with a galaxy of $m_{V}=20.9$ and redshift $z=0.566$ (di Serego Alighieri et al. 1994). The VLBI image at $2.3 \mathrm{GHz}$ (Fig. 5) shows a NorthSouth double structure quite symmetric; the peak regions are separated by $\sim 145$ mas $(\sim 0.54 \mathrm{kpc})$. Both components appear to be resolved and account for more than $95 \%$ of the whole flux density of $3.7 \mathrm{Jy}$ at this frequency. No "core" component is detected between the lobes $(5 \sigma=40 \mathrm{mJy})$ and no spectral information is available on the detected components.

1151-348 This object has been identified as a quasar with $m_{v}=17.8$ from WP85 and $z=0.258$ (Jauncey et al. 1978). Both 2.3 and $8.4 \mathrm{GHz}$ images are available (Fig. 6), showing two components with separation of 91 mas $(\sim 0.23 \mathrm{kpc})$ and $\mathrm{PA}=72^{\circ}$ and possibly some extended emission between them. The peak separation and orientation are identical (to within 0.5 mas) at both frequencies, unambiguously registering the two components.

Both lobes are less than $\sim 85$ mas $(\sim 0.2 \mathrm{kpc})$ in extent and the south-western one has an overall spectral index $(\alpha=-1.0)$ steeper than the north-eastern component $(\alpha=-0.6)$. About $70 \%$ of the total flux density is detected in the VLBI components and this fraction is the same at both frequencies. Thus the underlying undetected flux density has a spectral index ( -0.7) similar to the two aforementioned components, possibly 
Table 5. Size and flux density of the components in the images. Component angular sizes are estimated from the images (full width), except were otherwise noted.

\begin{tabular}{|c|c|c|c|c|c|c|c|c|c|}
\hline Source & Comp. & $\begin{array}{c}v \\
\mathrm{GHz}\end{array}$ & $\begin{array}{r}\theta_{1} \\
\text { mas }\end{array}$ & $\begin{array}{r}\theta_{2} \\
\text { mas }\end{array}$ & $\begin{array}{r}\text { Lsize1 } \\
\mathrm{pc}\end{array}$ & $\begin{array}{r}\text { Lsize2 } \\
\mathrm{pc}\end{array}$ & $\begin{array}{r}S_{\text {peak }} \\
\text { Jy/beam }\end{array}$ & $\begin{array}{r}S_{\text {tot. }} \\
\text { Jy }\end{array}$ & $\alpha_{2.3}^{8.4}$ \\
\hline $0023-263$ & NW & $\begin{array}{l}2.3 \\
8.4\end{array}$ & $\begin{array}{l}80 \\
40\end{array}$ & $\begin{array}{l}75 \\
23\end{array}$ & $\begin{array}{l}230 \\
110\end{array}$ & $\begin{array}{r}220 \\
65\end{array}$ & $\begin{array}{l}1.07 \\
0.41\end{array}$ & $\begin{array}{r}1.6 \\
0.65\end{array}$ & -0.7 \\
\hline & SE & $\begin{array}{l}2.3 \\
8.4\end{array}$ & $\begin{array}{r}100 \\
60\end{array}$ & $\begin{array}{l}90 \\
30\end{array}$ & $\begin{array}{l}290 \\
175\end{array}$ & $\begin{array}{r}260 \\
85\end{array}$ & $\begin{array}{r}0.33 \\
0.047\end{array}$ & $\begin{array}{r}1.3 \\
0.25\end{array}$ & -1.3 \\
\hline $0252-712$ & $\begin{array}{l}\text { North } \\
\text { South }\end{array}$ & 2.3 & $\begin{array}{l}110 \\
120\end{array}$ & $\begin{array}{l}90 \\
90\end{array}$ & $\begin{array}{l}400 \\
440\end{array}$ & $\begin{array}{l}330 \\
330\end{array}$ & $\begin{array}{l}1.14 \\
0.47\end{array}$ & $\begin{array}{l}2.1 \\
1.4\end{array}$ & \\
\hline $1151-348$ & $\begin{array}{l}\text { NE } \\
\text { SW }\end{array}$ & $\begin{array}{l}2.3 \\
8.4 \\
2.3 \\
8.4\end{array}$ & $\begin{array}{l}85 \\
40 \\
85 \\
35\end{array}$ & $\begin{array}{l}45 \\
30 \\
45 \\
20\end{array}$ & $\begin{array}{r}210 \\
100 \\
210 \\
90\end{array}$ & $\begin{array}{r}110 \\
75 \\
110 \\
50\end{array}$ & $\begin{array}{r}1.57 \\
0.437 \\
0.78 \\
0.12\end{array}$ & $\begin{array}{r}2.1 \\
0.94 \\
1.24 \\
0.36\end{array}$ & $\begin{array}{l}-0.6 \\
-1.0\end{array}$ \\
\hline $1306-095$ & $\begin{array}{l}\text { SE } \\
\text { NW }\end{array}$ & 2.3 & $\begin{array}{r}110 \\
80\end{array}$ & $\begin{array}{l}70 \\
50\end{array}$ & $\begin{array}{l}380 \\
275\end{array}$ & $\begin{array}{l}240 \\
170\end{array}$ & $\begin{array}{l}0.304 \\
0.107\end{array}$ & $\begin{array}{l}0.76 \\
0.15\end{array}$ & \\
\hline $1814-637$ & $\begin{array}{l}\text { North } \\
\text { South }\end{array}$ & 2.3 & $\begin{array}{r}90 \\
130\end{array}$ & $\begin{array}{l}80 \\
70\end{array}$ & $\begin{array}{r}70 \\
100\end{array}$ & $\begin{array}{l}65 \\
55\end{array}$ & $\begin{array}{r}1.58 \\
0.380\end{array}$ & $\begin{array}{l}2.8 \\
1.8\end{array}$ & \\
\hline $\begin{array}{l}1934-638^{a} \\
2135-209^{b}\end{array}$ & $\begin{array}{l}\text { NE } \\
\text { SW }\end{array}$ & 5.0 & $\begin{array}{l}25 \\
90\end{array}$ & $\begin{array}{r}9 \\
40\end{array}$ & $\begin{array}{l}100 \\
350\end{array}$ & $\begin{array}{l}350 \\
155\end{array}$ & $\begin{array}{r}1.02 \\
0.2\end{array}$ & $\begin{array}{r}1.1 \\
0.36\end{array}$ & \\
\hline
\end{tabular}

${ }^{a}$ Components and spectra for 1934-638 are given in Tzioumis et al. (1996).

${ }^{b}$ Component sizes from fitting Gaussians $(F W H M)$ since the beam is comparable to the source size and components cannot be estimated graphically.

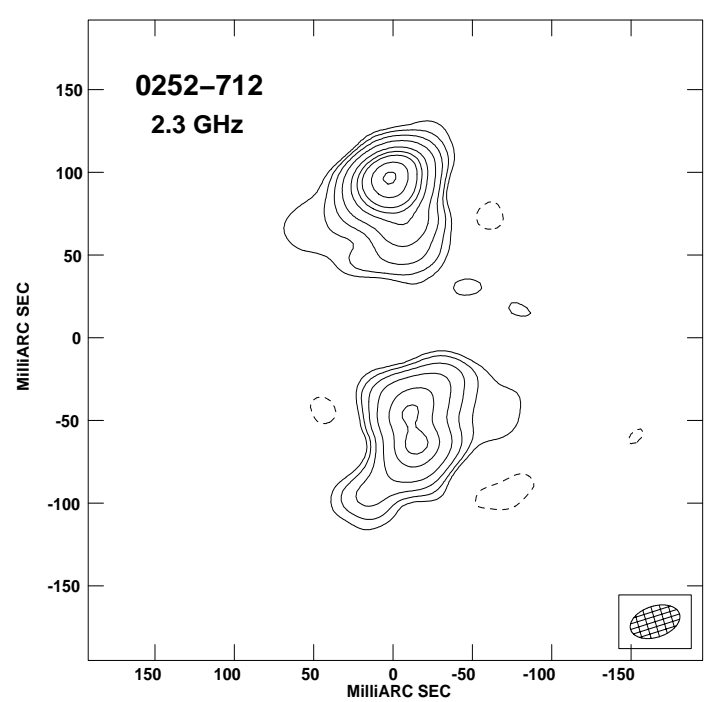

Fig. 5. PKS $0252-712$ at $2291 \mathrm{MHz}$ from the SHEVE array. The peak level is $1.14 \mathrm{Jy} /$ beam and contours are shown at $-1.5,1.5,3,6,12,24$, $36,48,72,96 \%$ of the peak.

indicating that this emission is simply resolved, diffuse extension of these lobes.

No flat spectrum component, possibly indicative of a core, is detected between the lobes, at the detection limit $(5 \sigma)$ of $\sim 20 \mathrm{mJy} /$ beam.
1306-095 It is identified with a galaxy of magnitude $m_{V}=20.5$ and redshift $z=0.464$ (di Serego Alighieri et al. 1994).

This source is at low absolute declination and the uv coverage is rather poor, which implies high sidelobe levels ( 50\%) and a low dynamic range image. The radio emission is dominated by two distinct components separated by about 373 mas $(\sim 1.3 \mathrm{kpc})$ in $\mathrm{PA} \sim-40^{\circ}$ (Fig. 7). The southern component is much stronger, accounting for more than $80 \%$ of the detected flux density. Only about $30 \%$ of the total flux density is detected by the VLBI components, indicating the presence of much diffuse emission completely resolved out by the VLBI baselines. It is likely that in Fig. 7 only the hot-spots of two mini-lobes are visible.

1814-637 It is identified with a galaxy of $m_{V}=18.0$ with $z=0.063$ by WP85. The VLBI image at $2.3 \mathrm{GHZ}$ shows the most complex structure of all sources in this sample (Fig. 8). The source still shows a basic double-lobed structure aligned almost North-South. However, the southern region is dominated by two components with similar brightness and much extended emission. The northern region shows a prominent bright component and extended emission, with possible North-South symmetrical extensions, but the overall extent of this component is still less than $\sim 90$ mas $(\sim 70 \mathrm{pc})$.

Just over $50 \%$ of the total flux density of the source is detected, indicating the presence of further, much more diffuse extended component. The overall source size is still less than about 0.5 arcsec and no arcsecond-scale components are detected with ATCA observations. 

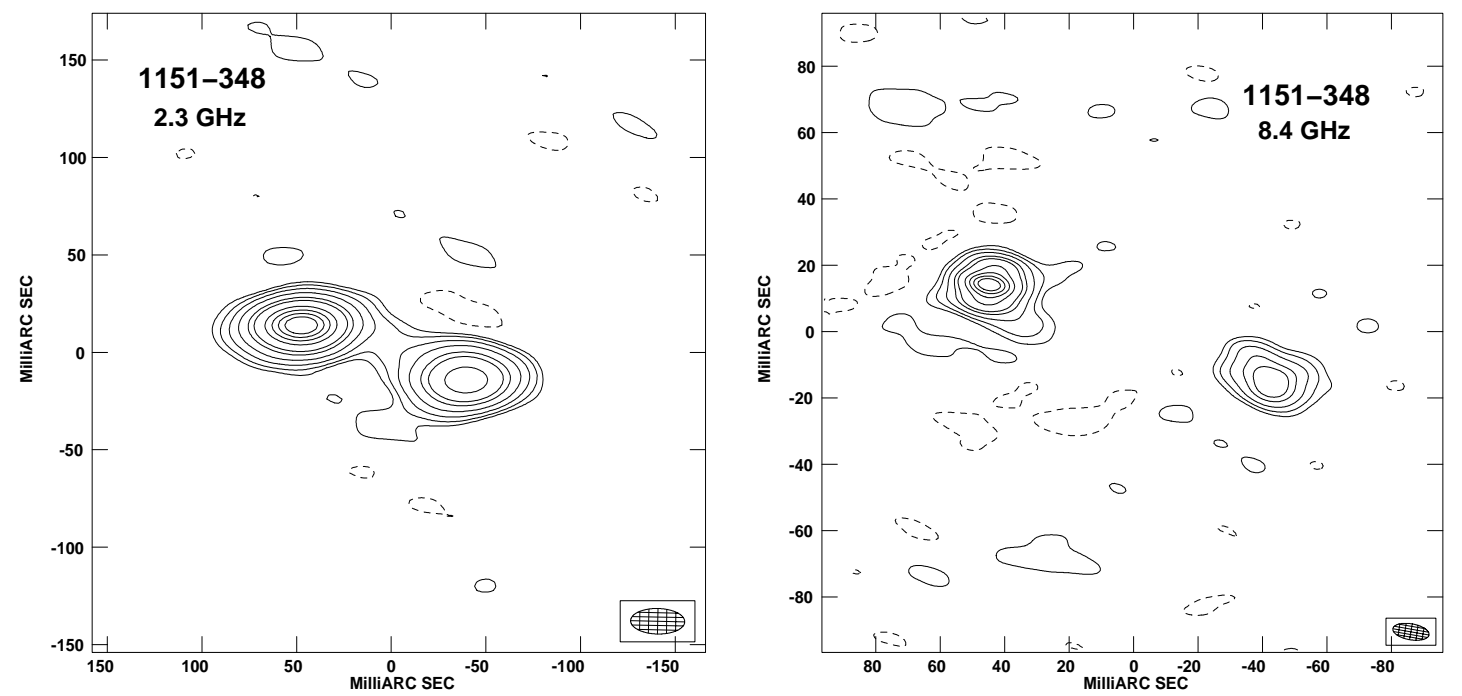

Fig. 6. (Left) PKS 1151-348 at $2291 \mathrm{MHz}$ from the SHEVE array. The peak level is $1.57 \mathrm{Jy} /$ beam and contours are shown at $-0.75,0.75,1.5$, $3,6,12,18,35,50,65,80 \%$ of the peak. (Right) PKS 1151-348 at $8421 \mathrm{MHz}$ from the SHEVE array. The peak level is $0.44 \mathrm{Jy} / \mathrm{beam}$ and contours are shown at $-1.5,1.5,3,6,12,18,35,50,65,80 \%$ of the peak.

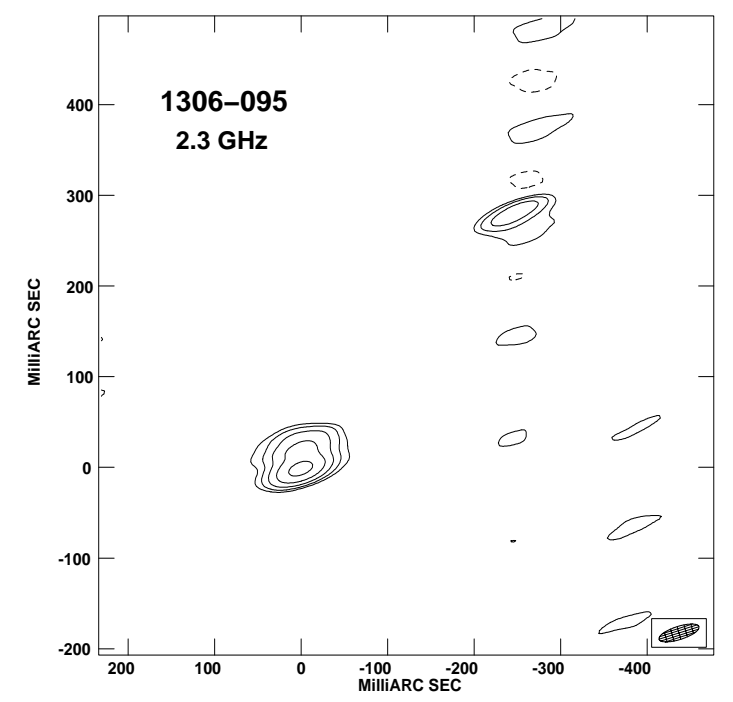

Fig. 7. PKS 1306-095 at $2291 \mathrm{MHz}$ from the SHEVE array. The peak level is $0.30 \mathrm{Jy} /$ beam and contours are shown at $-5,5,10,20,40,80 \%$ of the peak.

There is also a weak component between the two major lobes at the $5 \%$ level, which corresponds to a $15 \sigma$ detection. This can be just a peak in the underlying diffuse component or could indicate the presence of a core between the two lobes. VLBI observations at another frequency are needed to determine the spectrum of this component and decide whether it is the core.

1934-63 This compact radio source has been identified as a galaxy with $m_{V}=18.4$ by Kellerman (1966) and has redshift $z=0.183$ (Penston \& Fosbury 1978). A VLBI image at $2.3 \mathrm{GHz}$ from observations carried out in 1982 has been presented in Tzioumis et al. (1989) and preliminary images at 2.3, 4.8 and $8.4 \mathrm{GHz}$ have also been presented elsewhere (King 1994; Tzioumis et al. 1996). The knowledge about the pc-scale structure of this source is summarised in Fig. 10. The source appears as a very compact double, with component separation of 42 mas $(\sim 0.084 \mathrm{kpc})$ in PA $89^{\circ}$, and this separation has not changed for over 15 years. As reported in Tzioumis et al. (1996), this yield to an upper limit to the expansion velocity of $0.2 c$. The total flux density of the source is detected by the VLBI components and there is no definite detection of a flat-spectrum core component at a level of a few $\%$ of the peak at any frequency.

2135-209 This source is a galaxy with $m_{v}=19.4$ and $z=0.635$ (WP85 and di Serego Alighieri et al. 1994).

No VLBI image exists for this source and here we present a MERLIN 5 GHz image (Fig. 9). The source shows a double lobed very asymmetric structure; the resolution of MERLIN at this southern declination is poor $(163 \times 34$ mas in the case of 2135-209) to allow further consideration on the morphology. Because of the limited resolution compared to the source extension, the component parameters were derived by means of Gaussian fitting. The North-East dominant component is marginally resolved and accounts for more than $80 \%$ of the detected flux density. The total flux density of about $1.5 \mathrm{Jy}$ was detected in the MERLIN image, indicating no extra extended components exist.

From one-baseline VLBI observations at $2.3 \mathrm{GHz}$ it is possible to fit models to the double structure. The strong northeast component is unresolved while the south-west component is extended. The separation between the peaks in the two lobes is well determined at 167 mas $(\sim 0.65 \mathrm{kpc})$, at a position angle of $52^{\circ}$, close to the position angle and separation determined from the MERLIN image. About $85 \%$ of the total flux density is detected on this VLBI baseline. 


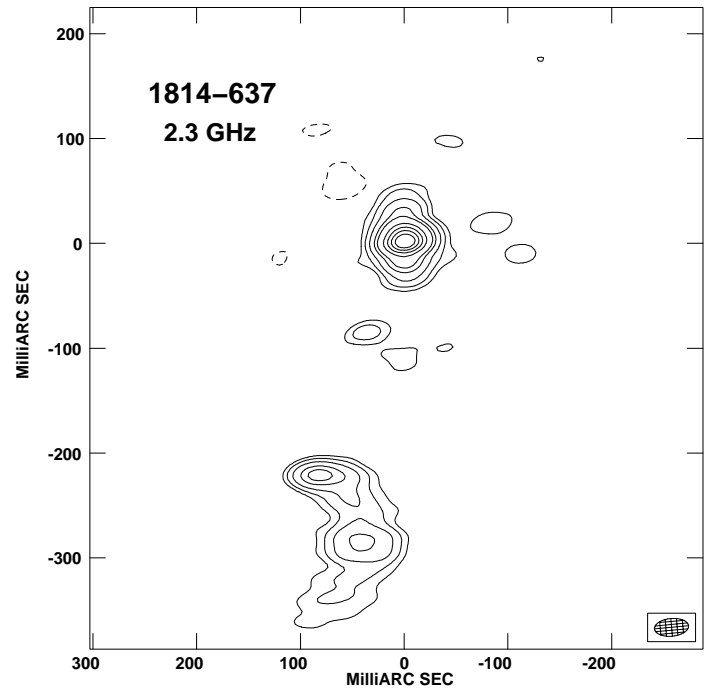

Fig. 8. PKS $1814-637$ at $2291 \mathrm{MHz}$ from the SHEVE array. The peak level is $1.7 \mathrm{Jy} /$ beam and contours are shown at $-1.5,1.5,3,6,12,18$, $35,50,65,80 \%$ of the peak.

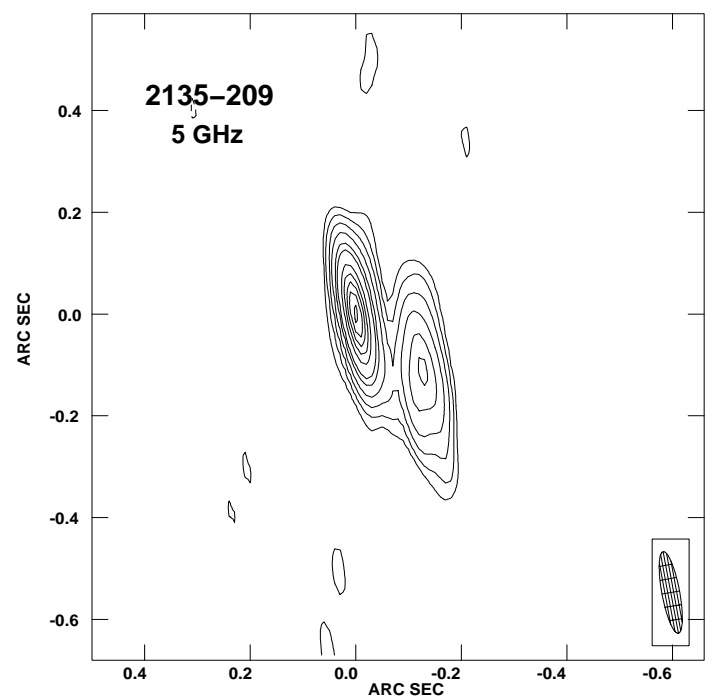

Fig. 9. PKS 2135-209 at $4993 \mathrm{MHz}$ from the MERLIN array. The peak level is $1.02 \mathrm{Jy} /$ beam and contours are shown at $-0.75,0.75,1.5,3,6$, $12,18,35,50,65,80,95 \%$ of the peak.

\section{Discussion}

The main aim of these high resolution observations was the morphological classification of a group of CSS sources found in a larger, flux-limited sample mainly consisting of extended radio sources. Like the majority of CSS sources studied in previous works, the objects observed here show mainly a doublelobed structure, often asymmetric. The structures revealed by these observations are generally resolved and they may well represent the lobes of small-sized powerful radio sources. In fact, for the two sources observed at both 8.4 and $2.3 \mathrm{GHz}$, only steep spectrum components have been detected. The present observations do not reveal any definite core candidates, but in one case (1814-637) there is a weak third component between the lobes which could be suggested as a possible core. Weak tails are found pointing towards the source centre in a few cases, further suggesting that we are seeing two-sided emission from lobes and possibly hot-spots.

Generally, these images do not show many highly distorted or complex structures, which could be ascribed either to the interaction between the radio emitting plasma and the ambient medium or to projection effects. Even in the object which shows the most complex structure (1814-637), the basic double-lobed morphology is still clearly evident. Source linear sizes are in the range $0.08-2.9 \mathrm{kpc}$, and hence all sources in this sample can be classified as compact symmetric (CSO) or medium-sized symmetric (MSO) objects if we assume that an undetected core sits between the lobes revealed by the present observations.

Dallacasa et al. (1995) have investigated a sample of northern CSS sources (using MERLIN and EVN) selected from the Peacock \& Wall catalogue (1981). As their sample and the present sample have the same selection and observing frequencies, it is expected that sources in both samples should have, on average, similar characteristics. CSS sources selected from the Wall \& Peacock sample tend to have a higher turnover frequency and smaller size (e.g. Dallacasa et al. 1995) because of the higher selection frequency (i.e. $2.7 \mathrm{GHz}$ ) compared with the 3CR sample (178 MHz).

The size of the sources in the present sample range between $\sim 0.08$ and $\sim 2.9 \mathrm{kpc}$, similar to the range found in the sample studied in Dallacasa et al. (1995), and smaller than the 3CR samples (Fanti et al. 1990 and references therein). Turnover frequencies are in the range around $0.1-0.2 \mathrm{GHz}$ or below, with the exception of 1934-63 that peaks at $\sim 1.4 \mathrm{GHz}$. As expected from the correlation between the linear size of the source and the turnover frequency (Fanti et al. 1990), the smallest source in our sample is the GPS source 1934-638, that has the turnover at higher frequency.

Dallacasa et al. (1995) also find that most of galaxies their PW CSS sample show weak core candidates at $\lesssim 2 \%$ of the total flux density, in contrast with the 3CR samples, where, however, the dynamic range was smaller and comparable to these achieved for most of the sources presented in this paper.

Of the seven CSS/GPS objects presented in this paper, four have been searched for HI absorption (0023-26, 1814-63, 1934-63 and 2135-20). The results have been summarised in Morganti et al. (2001) while the preliminary results from new VLBI observations for 1814-63 have been presented in Morganti et al. (2000).

Of the four objects, three are detected. The only one undetected is 2135-209, but this result is not too surprising given that it is classified as broad-line galaxy. It is interesting, however, that the most impressive HI absorption is detected in the object with the most complex radio structure in our small sample, 1814-63. The deep HI absorption appears extended and cover most of the source with the exception of the southern part of the southern lobe. The peak optical depth goes from $\sim 30 \%$ in the southern lobe to $\sim 10 \%$ in the northern region. The component with lower optical depth (ranging between 2 and 4\%) is observed only against the northern region. Using the available systemic velocity $\left(V_{\text {hel }}=19350 \mathrm{~km} \mathrm{~s}^{-1}\right.$, Morganti et al. 2001), most of the H I absorption appears to be blueshifted. This can be explained by the presence of extended gas, 

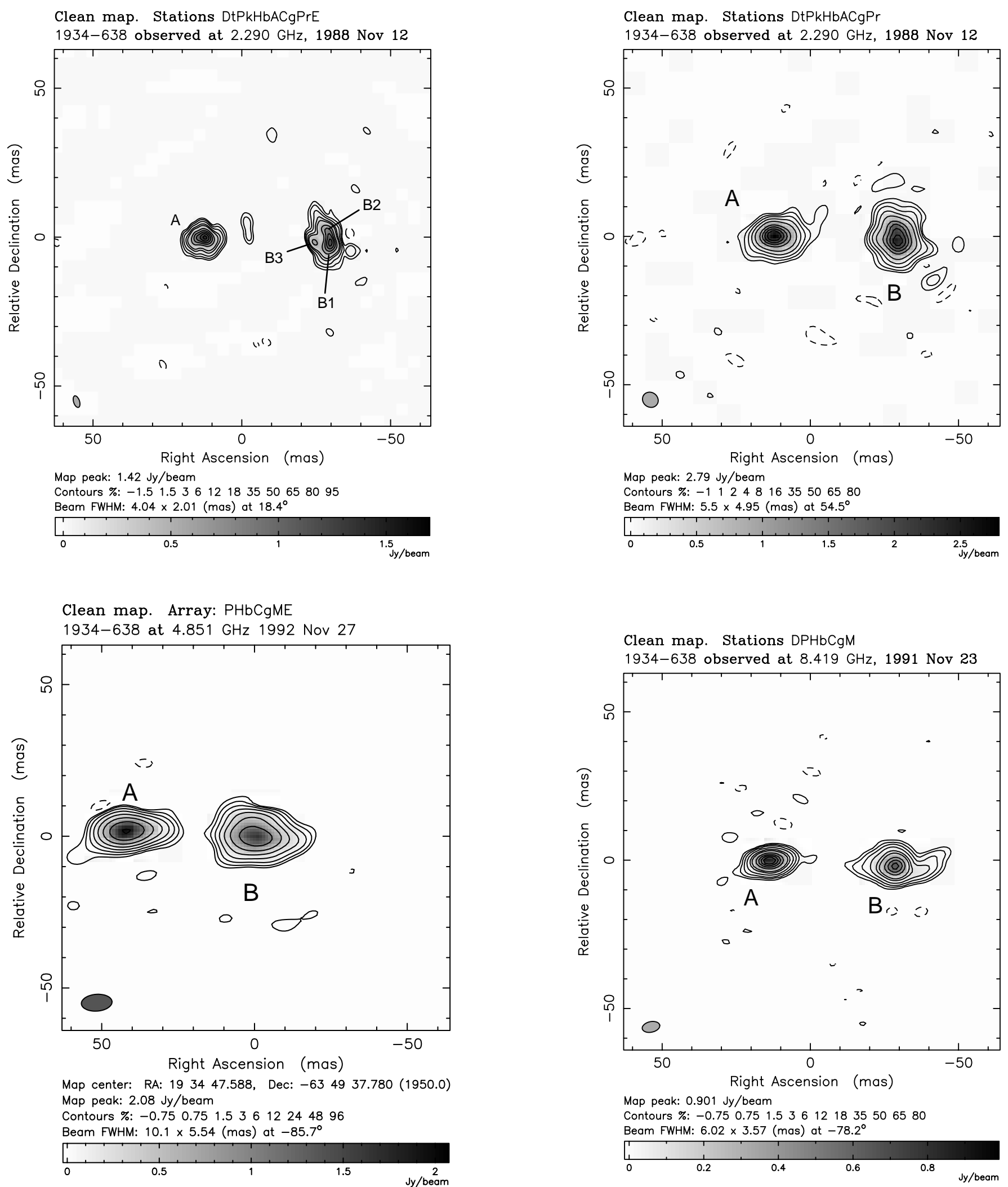

Fig. 10. VLBI images of PKS 1934-638 at 2.3, 4.8 and $8.4 \mathrm{GHz}$ (from Tzioumis et al. 1997). The top-right image is at a higher resolution because it includes Hartebeesthoek data. The other three images have approximately the same resolution of about 5 mas.

possibly surrounding the lobes, interacting/expanding with the radio plasma. Therefore, in the case of 1814-63, part of the radio morphology could be affected by a strong interaction with the ISM. 
Overall, the properties of the sources discussed here are consistent with the results from the analysis of other samples of CSS sources. In a forthcoming paper we will discuss their properties in wavebands different from radio, compare them with those of extended radio sources and verify whether they support and "youth" scenario.

\section{References}

Axon, D. J., Capetti, A., Fanti, R., et al. 2000, AJ, 120, 2284

Bicknell, J., Dopita, M., \& O’Dea, C. 1997, ApJ, 485, 112

Clark, B. G. 1973, Proc. IEEE, 61, 1242

Conway, J. E. 1996, in The second Workshop on Gigahertz Peaked Spectrum and Compact Steep Spectrum Radio Sources, ed. I. A. G. Snellen, R. T. Schilizzi, H. J. A. Röttgering, \& M. N. Bremer (M.N. Publ JIVE, Leiden), 198

Dallacasa, D., Fanti, C., Schilizzi, R. T., \& Spencer, R. E. 1995, A\&A, 295, 27

Fanti, C., Pozzi, F., Fanti, R., et al. 2000, A\&A, 358, 499

Fanti, R., Fanti, C., Schilizzi, R. T., et al. 1990, A\&A, 231, 333

Fanti, C., Fanti, R., Dallacasa, D., et al. 1995, A\&A, 302, 317

Fanti, C. 2000, Proc. of the 5th European VLBI Network Symp., ed. J. E. Conway, A. G. Polatidis, R. S. Booth, \& Y. M. Pihlström, ISBN 91-631-0548-9, 73

Gelderman, R., \& Whittle, M. 1994, ApJS, 91, 491

Jauncey, D. L., Wright, A. E., Peterson, B. A. \& Condon, J. 1978, ApJ, 219, L1

Jauncey, D. L., \& the SHEVE Team 1994, in Very high angular resolution imaging, IAU158, ed. J. G. Robertson, \& W. J. Tango (Dordrecht: Kluwer), 131

Kellerman, K. I. 1966, ApJ, 146, 621

King, E. 1994, Ph.D. Thesis Univ. of Tasmania

Morganti, R., Killeen, N. E. B., \& Tadhunter, C. N. 1993, MNRAS, 263,1023

Morganti, R., Oosterloo, T., Tadhunter, C. N., et al. 2001, MNRAS, 323,331

Morganti, R., Oosterloo, T. A., Reynolds, J., Tadhunter, C. N., \& Migenes, V. 1997, MNRAS, 284, 541

Morganti, R., Oosterloo, T. A., Tadhunter, C. N., et al. 1999, A\&AS, 140,355
Morganti, R., Oosterloo, T., Tadhunter, C. N., et al. 2000, Proc. of the 5th European VLBI Network Symp., ed. J. E. Conway, A. G. Polatidis, R. S. Booth, \& Y. M. Pihlström, ISBN 91-6310548-9, 111 [astro-ph/0010482]

Murgia, M., Fanti, C., Fanti, R., et al. 1999, A\&A, 345, 769

O’Dea, C. P., Baum, S. A., \& Stanghellini, C. 1991, ApJ, 380, 66

O’Dea, C. P. 1998, PASP, 110, 493

O'Dea, C. P., de Vries, W. H., Koekemoer, A. M., et al. 2002, AJ, 123, 2330

Owsianik, I., \& Conway, J. E. 1998, A\&A, 337, 69

Padovani, P., Morganti, R., Siebert, J., Cimatti, A., \& Vagnetti, F. 1999, MNRAS, 304, 829

Peck, A. B., \& Taylor, G. B. 2001, ApJ, 554, L147

Penston, M. V., \& Fosbury, R. A. E. 1978, MNRAS, 183, 479

Pihlström, Y. M. 2001, Ph.D. Thesis, Chalmers University \& Onsala Observatory

Preston \& the SHEVE Team, 1993, in Sub-arcsecond Radio Astronomy, ed. R. J. Davis, \& R. S. Booth (Cambridge University Press), 428

di Serego Alighieri, S., Danziger, J. I., Morganti, R., \& Tadhunter, C. 1994, MNRAS, 269, 998

de Vries, W., O’Dea, C. P., Baum, S. A., et al. 1998, ApJ, 503, 138

Sanghera, H. S. 1993, Ph.D. Thesis, Univ. of Manchester

Shepherd, M. C., Pearson, T. J., \& Taylor, G. B. 1994, BAAS, 26, 987

Siebert, J., Brinkmann, W., Morganti, R., et al. 1996, MNRAS, 279, 1331

Silk, J., \& Rees, M. J. 1998, A\&A, 331, L1

Spencer, R. E., Schilizzi, R. T., Fanti, et al. 1991, MNRAS, 250, 225

Tadhunter, C. N., Morganti, R., di Serego Alighieri, S., Fosbury, R. A. E., \& Danziger, I. J. 1993, MNRAS, 263, 999

Tadhunter, C. N., Shaw, M., \& Morganti, R. 1994, MNRAS, 271, 807

Tadhunter, C. N., Morganti, R., Robinson, A., et al. 1998, MNRAS, 298, 1035

Trussoni, E., Vagnetti, F., Massaglia, S., et al. 1999, A\&A, 348, 437

Tzioumis, A. K., King, E. A., Jauncey, D. L., et al. 1996, in The second Workshop on Gigahertz Peaked Spectrum and Compact Steep Spectrum Radio Sources, ed. I. A. G. Snellen, R. T. Schilizzi, H. J. A. Röttgering, \& M. N. Bremer (M.N. Publ JIVE, Leiden), 58

Venturi, T., Morganti, R., Tzioumis, A., \& Reynolds, J. 2000, A\&A, 363,84

Wall, J., \& Peacock, J. 1985, MNRAS, 216, 173 